\title{
Chaos Control and Noise Suppression in External-Cavity Semiconductor Lasers
}

\author{
Noriyuki Kikuchi, Yun Liu, and Junji Ohtsubo, Member, IEEE
}

\begin{abstract}
Feedback-induced chaos and intensity noise enhancement in a laser diode with external optical feedback are studied by computer simulations. The enhancement of relative intensity noise (RIN) that is often observed in experiments is considered as a result of the feedback-induced deterministic chaos and the intensity noise suppression is treated from the viewpoint of chaos control. Especially, the conventional noise suppressing technique known as a high-frequency injection modulation is turned into a problem of stabilizing chaos through parameter modulations. We developed an analytical method which allows to optimize the modulation frequency from the linear stability analysis of the dynamical model that describes the laser diode with external feedback. The robustness of the modulation with respect to the modulation frequency and depth is verified and the results suggest the feasibility of applying our method to actual noise suppression. The RIN in the low-frequency region (up to $100 \mathrm{MHz}$ is shown to be reduced to the solitary laser level when the feedback-induced chaos is effectively controlled with the optimized modulation frequency.
\end{abstract}

Index Terms - Chaos control, external feedback, highfrequency modulation, noise suppression, semiconductor laser.

\section{INTRODUCTION}

$\mathbf{T}$ HE PERFORMANCE of a semiconductor laser in optical data recording or optical communication systems is crucially influenced by the amount of optical feedback from the external reflectors such like optical disk surface or fiber connectors. While weak feedback (up to approximately $-20 \mathrm{~dB}$ in an external-cavity length of $5-10 \mathrm{~cm}$ ) results in linewidth narrowing and improves frequency stability, moderate to strong feedback levels will usually cause a sudden line width broadening known as "coherence collapse," which is accompanied with the intensity noise enhancement [1]. To overcome such feedback-induced unfavorable effects, various methods have been proposed including the high-frequency injection (HFI) technique [2], [3] that modulates the laser injection current with periodic signals whose frequency is much higher than the data rate. Experimental results have demonstrated that the RIN level can be dramatically reduced by appropriate modulations.

Manuscript received December 28, 1995; revised September 30, 1996. This work was supported by the Research Foundation for the Electrotechnology of Chubu.

N. Kikuchi and J. Ohtsubo are with the Faculty of Engineering, Shizuoka University, Hamamatsu 432 , Japan.

Y. Liu is with the Graduate School of Electronic Science and Technology, Shizuoka University, Hamamatsu 432, Japan.

Publisher Item Identifier S 0018-9197(97)00362-X.
Recently, several groups [4], [5] proposed to treat the problem of the HFI technique from a chaos controlling point of view. According to their conclusions, the feedback-induced intensity noise enhancement is considered as a result of feedback-induced deterministic instabilities, i.e., chaos. Therefore, one can realize the suppression of the feedback induced intensity noise provided the feedback-induced chaos is effectively controlled. Meanwhile, chaos control though direct periodic modulation or parameter perturbation has been stud ied in various numerical and experimental systems [6]-[10]. However, there has been a lack of effective means so far for determining the modulation frequency which is a key point in performing the modulation. In our previous work [5], we proposed a new method to optimize the modulation frequency from the linear stability analysis of the dynamical model of the system. Our method gives an analytical means to determine the modulation frequency. The numerical results demonstrated that the feedback-induced chaos can be stabilized to a limit cycle with a small modulation depth (the laser is always above the threshold during the modulation) by choosing the modulation frequency equal to one of the linear modes.

The purpose of this paper is to present a detailed study on the chaos control and the theoretical background of the noise reduction via the HFI technique in the external-cavity semiconductor laser. Unlike the conventional HFI modulation where the modulation depth is large enough and the laser is driven below threshold over a part of the modulation cycle, the modulation depth used in our work is restricted to a small range to let the laser operate above threshold. Another notable feature of the method is that the modulation frequency is optimized analytically based on the model of the laser diode with external feedback. By seanning the modulation frequency, we find that, around analytically obtained frequencies, there exist frequency "windows" where chaos can be stabilized to limit cycles with a small modulation depth. To reveal the relation between the chaos and the noise reduction, we include the Langevin noises in our model and calculate the RIN spectra. The results show that the RIN in the low-frequency region can be reduced to the solitary laser level when chaos is controlled.

The numerical calculations are restricted to low feedback level $(-45$ to $-30 \mathrm{~dB}$ in power) and weak modulation (never exceeds the threshold). The numerical results demonstrated that, as increasing the feedback level, the laser undergoes a quasi-periodic route to chaos as reported in other experiments [11], [12]. The oscillation frequency is dominated by the relaxation frequency of the laser rather than the external-cavity 


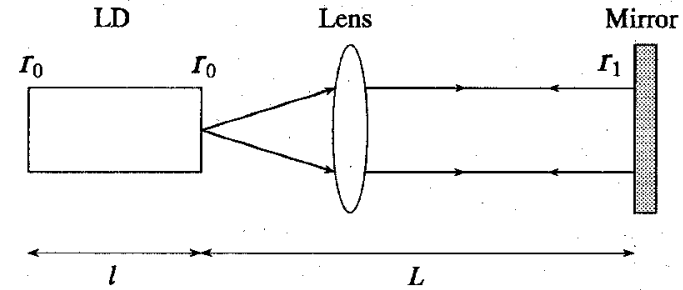

Fig. 1. Schematic illustration of a laser diode with external optical feedback.

beating frequency $f_{\text {ext }}=c / 2 L$, where $L$ is the optical length of the external cavity. It should be noted that neither the subharmonic bifurcations and external-cavity mode-locking which occurred for strong feedback (about $-10 \mathrm{~dB}$ in power) [13] nor the modulation instabilities which were observed for large modulation [14] has been observed for the weak feedback and low modulation depth. For this reason, we employed a single-mode model, instead of a multimode one used in [4], to describe dynamical and noise behaviors of the laser diode. The linear stability analysis conducted in this paper can also be extended to multimode model, but the applicability should be restricted to the case of small modulation depth since otherwise the strong modulation would rather dominate dynamics of the laser.

This paper is organized as follows. Section II gives a brief description about the dynamical model of the laser diode with external optical feedback, the bifurcation routes with respect to the feedback parameters, and the linear stability analysis. Section III studies the modulation results of the laser diode for various modulation frequencies that are chosen based on the mode distribution obtained from the linear stability analysis. The dynamical range for the modulation amplitude and frequency is investigated to guarantee the robustness of the chaos stabilization via the injection modulation. In Section IV, spontaneous emission and shot noises are generated through Langevin terms in the rate equations and the RIN level is calculated for both the controlled and uncontrolled situations. Particular attention is paid to the variation of the RIN level when chaos is stabilized to limit cycles. Referring to actual situations such as the noise suppression in optical data recording systems, we investigate the RIN suppression in low-frequency region up to $100 \mathrm{MHz}$. Section $\mathrm{V}$ concludes the paper with discussions on the comparison of our numerical results with some related experiments and the connection between the mechanism of the direct modulation and the conventional chaos control algorithms, i.e., the OGY and occasional proportional feedback (OPF) control techniques.

\section{Numerical Model AND LineAR STABILITy ANALYSIS}

Fig. 1 shows the schematic of a laser diode with external optical feedback. The internal amplitude reflectivity $r_{0}$ is assumed for both the front and rear facets of the laser diode. The light beam is reflected by an external mirror located at a distance $L$ from the front facet of the laser diode cavity. The fraction of the electric amplitude of the output light coupled into the cavity is denoted as $r_{1}$, which includes the amplitude reflectivity of the external mirror, the propagation losses in the
TABLE I

Some Parameter Values for the Laser DiOde USED IN the Numerical Simulations

\begin{tabular}{cll}
\hline Symbol & Parameter & Value \\
\hline$G_{N}$ & Gain coefficient & $8.4 \times 10^{-13} \mathrm{~m}^{3} \mathrm{~s}^{-1}$ \\
$\alpha$ & Linewidth enhancement factor & 3 \\
$r_{0}$ & Facet reflectivity & 0.556 \\
$N_{t h}$ & Carrier density at threshold & $2.018 \times 10^{24} \mathrm{~m}^{-3}$ \\
$N_{o}$ & Carrier density at transparency & $1.400 \times 10^{24} \mathrm{~m}^{-3}$ \\
$\tau_{s}$ & Life time of carrier & $2.04 \mathrm{~ns}$ \\
$\tau_{p}$ & Life time of photon & $1.927 \mathrm{ps}$ \\
$\tau_{t n}$ & Round trip time in laser cavity & $8 \mathrm{ps}$ \\
$V$ & Volume of active region & $1.2 \times 10^{-16} \mathrm{~m}^{3}$ \\
\hline
\end{tabular}

external optical path, and the coupling efficiency through the lens, the facet, and etc.

The dynamics of a single-mode laser diode with weak to moderate feedback can be modeled by the noise driven equations for the amplitude $E_{0}(t)$ and the phase $\phi(t)$ of the electric field and the average carrier density $N(t)$ in the active region [11], [12], [15]

$$
\begin{aligned}
\frac{d E_{0}(t)}{d t}= & \frac{1}{2}\left\{G_{N}\left[N(t)-N_{\mathrm{th}}\right]-\frac{1}{\tau_{p}}\right\} E_{0}(t) \\
& +\frac{\kappa}{\tau_{\mathrm{in}}} E_{0}(t-\tau) \cos \Delta(t)+\frac{R_{\mathrm{sp}}}{2 V E_{0}(t)}+F_{e}(t) \\
\frac{d \phi(t)}{d t}= & \frac{\alpha}{2} G_{N}\left[N(t)-N_{\mathrm{th}}\right] \\
& -\frac{\kappa E_{0}(t-\tau)}{\tau_{\mathrm{in}} E_{0}(t)} \sin \Delta(t)+\frac{F_{\phi}(t)}{E_{0}(t)} \\
\frac{d N(t)}{d t}= & J-\frac{N(t)}{\tau_{s}} \\
& -G_{N}\left[N(t)-N_{\mathrm{th}}\right]\left|E_{0}(t)\right|^{2}+F_{N}(t)
\end{aligned}
$$

with

$$
\Delta(t)=\omega_{0} t+\phi(t)-\phi(t-\tau)
$$

Here, $E_{0}(t)$ is rescaled so that $\left|E_{0}(t)\right|^{2}$ corresponds to the total photon number in the laser diode cavity, $G_{N}$ is the modal gain coefficient, $N_{0}$ is the carrier density at transparency, $N_{\mathrm{th}}$ is the threshold carrier density, $\omega_{0}$ is the angular frequency of the solitary laser, and $F_{e}, F_{\phi}, F_{N}$ are Langevin noise terms. Also, $\tau_{p}$ is the photon lifetime, $\tau_{s}$ is the carrier lifetime, $\tau_{\text {in }}=2 \eta l / c$ is the optical round-trip time, $\tau=2 L / c$ is the external-cavity round-trip time, $\alpha$ is the linewidth enhancement factor, $J$ is the injection current density, and $R_{\mathrm{sp}}$ represents the contribution of the spontaneous emission to the lasing mode. The feedback parameter $\kappa$ is given by

$$
\kappa=\left(1-r_{0}^{2}\right) \frac{r_{1}}{r_{0}} \text {. }
$$

We neglect the small contribution from spontaneous emission to the lasing mode. This factor is evaluated to be much smaller (less than one percent) than the feedback term (at $r_{1}=1 \%$ ) and negligible compared with both the gain and 

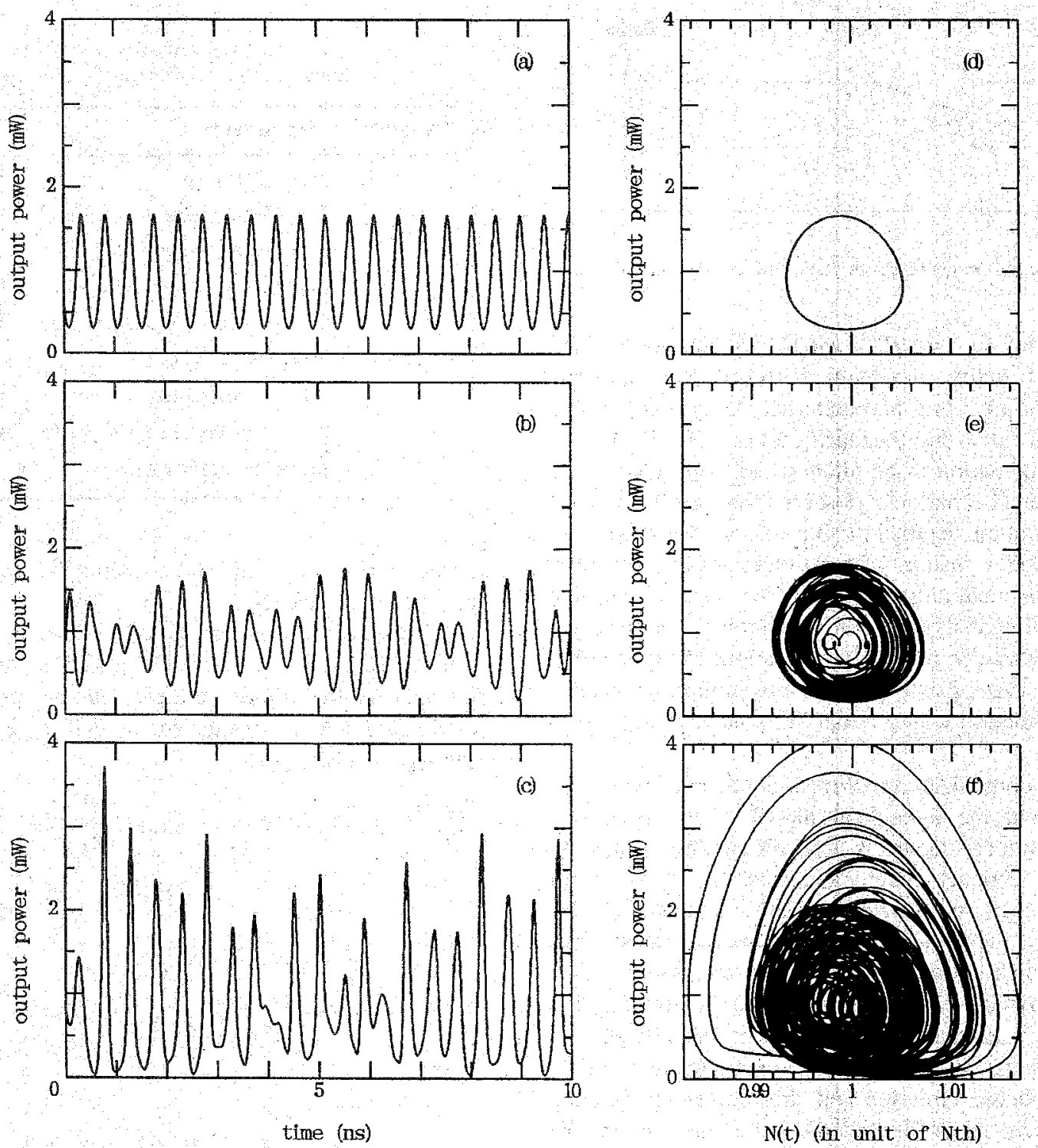

Fig. 2. Temporal variations and their corresponding phase portraits for $J=1.2 J_{\mathrm{th}}, L=9 \mathrm{~cm}$, and (a) and (d) periodic state at $r_{1}=1 \%$, (b) and (e) quasi-periodic state at $r_{1}=1.5 \%$, and (c) and (f) chaos at $r_{1}=2 \%$.

loss factors. In our calculations with low feedback level and weak modulation, it yields negligible influence $(<1 \%)$ to the dynamics of laser output, the chaos stabilization frequency windows, and the noise reduction. Another effect of the spontaneous emission, i.e, the spontaneous emission noise [16], is included in Langevin terms and its influences on RIN are discussed in Section IV. Some numerical values adopted for the various parameters, as appropriate for a 780 nm AlGaAs semiconductor laser likely to be used in an optical recording system, are given in Table I [1]-[3].

Equations (1)-(3) can be written in a general form

$$
\frac{d \mathbf{X}(t)}{d t}-f(\mathbf{X}(t))+\kappa \cdot g(\mathbf{X}(t), \mathbf{X}(t-\tau))+\mathbf{F}(t)
$$

where $\mathbf{X}(t)=\left(E_{0}(t), \phi(t), N(t)\right), \mathbf{F}(t)=\left(F_{e}(t), F_{\phi}(t)\right.$, $\left.F_{N}(t)\right), f$ is a nonlinear vector function describing a solitary laser, and $\kappa \cdot g$ is the nonlinear feedback term with the delay time $\tau$ and the strength $\kappa$. It is reasonable to take $\kappa$ as a control parameter in deternining the dynamical behaviors of the extemal-cavity laser diode.

We have numerically calculated (1) (3) by employing a fourth order Runge-Kutta algorithm and investigated various cases for steady, periodic, quasi-periodic, and chaotic states. In order to separate deterministic chaos from stochastic noise, we did not include noises in the present simulations. The noise effects will be considered in Section IV with the RIN evaluation. Fig. 2(a)-(c) shows the results of temporal variations for $J=1.2 J_{\mathrm{th}}$ and $L=9 \mathrm{~cm}$, where $J_{\mathrm{th}}=N_{\mathrm{th}} / \tau_{s}$ is the current density at the threshold. As the external reflectivity $r_{1}$ is increased from $0.5 \%$ to $2 \%$, we observed a continuous bifurcation from stationary state to periodic [Fig. 2(a)], quasi-periodic [Fig. 2(b)], and finally chaotic [Fig. 2(c)] states. Their corresponding phase portraits, namely, limit cycle, torus, and chaotic attractor are shown in Fig. 2(d), (e), and (f), respectively. Note that the photon number calculated in the simulations is converted to the light power emitted from the 


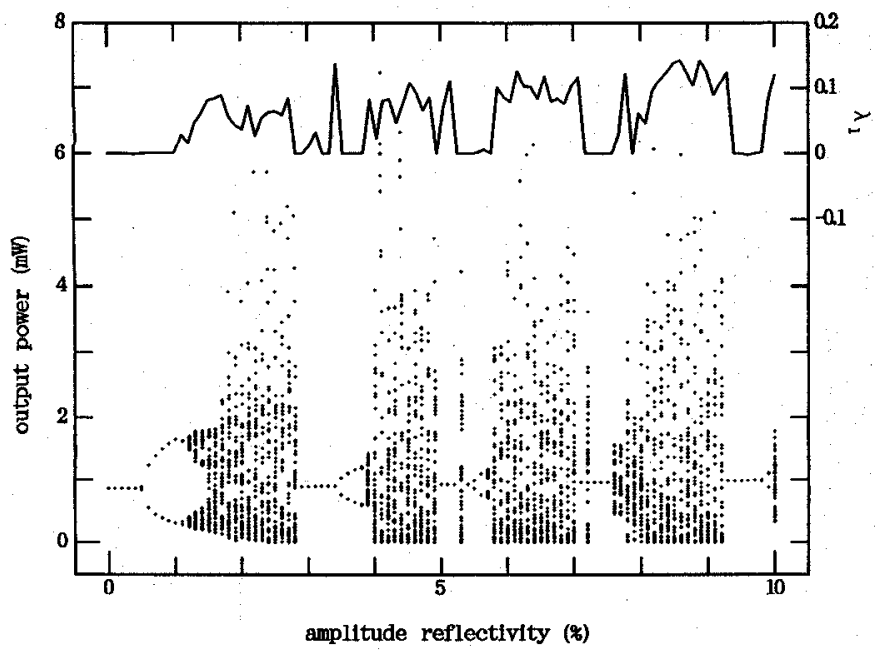

Fig. 3. Bifurcation diagram and the largest Lyapunov exponent $\lambda_{1}$ as a function of external reflectivity for $J=1.2 J_{\mathrm{th}}, L=9 \mathrm{~cm} . r_{1}$ is varied from 0 to $10 \%$.

rear facet of the laser diode in the figure. The bifurcation scenario repeats for further increasing of $r_{1}$. Such quasiperiodic bifurcation route has also been investigated both experimentally and numerically in other works [11], [12] with similar feedback conditions. Fig. 3 shows the bifurcation diagram for the range of $r_{1} \in[0-10 \%]$. Each dot in Fig. 3 represents the peak value of the output power. Lyapunov spectra are also calculated from the time series and the largest Lyapunov exponent $\lambda_{1}$ is shown to help recognizing chaos $\left(\lambda_{1}>0\right)$ and periodic states $\left(\lambda_{1} \approx 0\right)$. We can also obtain the similar results as shown in Fig. 3 by varying other parameters like injection current or external-cavity length, though they are not shown here.

To control chaos as shown in Fig. 2(c), the HFI technique which modulates the laser diode injection current is often employed. The algorithm is written as

$$
J=J_{b}[1+\xi \sin (2 \pi \nu t)]
$$

where $J_{b}$ is the bias current density, $\xi$ is the modulation depth, and $\nu$ is the modulation frequency. In performing the above modulation, the modulation frequency turns out to be an important factor. In our previous work [5], we proposed to choose the modulation frequency based on the linear mode distribution that is obtained from the linear stability analysis of the dynamic model. Here, we briefly review the linear stability analysis and mode distributions [17].

To start with, we calculate the steady-state solutions to (1)-(3) as

$$
\begin{aligned}
E_{0}(t) & =E_{s} \\
\phi(t) & =\left(\omega_{s}-\omega_{0}\right) t \\
N(t) & =N_{s} .
\end{aligned}
$$

By inserting (8) to (1)-(3), we obtain the following equations for the steady-state solutions:

$$
\omega_{\mathrm{s}}-\omega_{0}=-\frac{\kappa}{\tau_{\text {in }}}\left[\alpha \cos \left(\omega_{\mathrm{s}} \tau\right)+\sin \left(\omega_{\mathrm{s}} \tau\right)\right]
$$

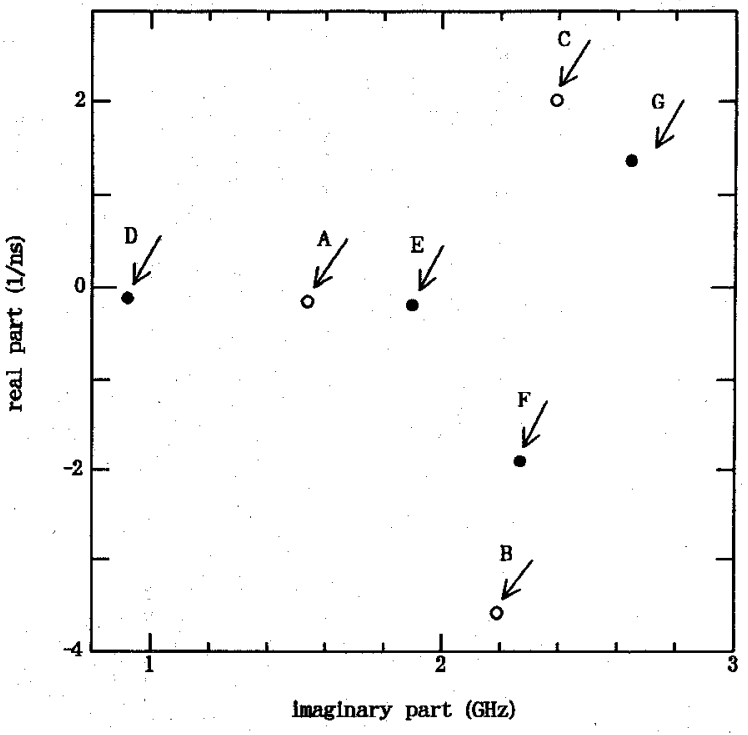

Fig. 4. Mode distributions for two typical chaotic outputs. White circles are for $J=1.2 J_{\mathrm{th}}, L=9 \mathrm{~cm}$, and $r_{1}=2 \%$ and black circles are for $J=1.3 J_{\mathrm{th}}, L=15 \mathrm{~cm}$, and $r_{1}=2.5 \%$.

$$
\begin{aligned}
G_{N}\left(N_{s}-N_{\mathrm{th}}\right) & =-\frac{2 \kappa}{\tau_{\mathrm{in}}} \cos \left(\omega_{s} \tau\right) \\
G_{N}\left(N_{s}-N_{0}\right) E_{s}^{2} & =J_{b}-\frac{N_{s}}{\tau_{s}} .
\end{aligned}
$$

In general, there exist a number of solutions for $\omega_{s}$ even for a fixed set parameters. Among them, we choose one that results in the maximum gain as a steady-state solution for the angular frequency. The steady-state carrier density and field amplitude are uniquely determined from (10) and (11) for a specific value of $\omega_{s} \tau$.

To analyze the stability to a small perturbation from the stationary state, we assume three variables oscillate around their stationary states in the form

$$
x(t)=x_{s}+\delta x \exp (\gamma t) \quad\left(x=E_{0}, N, \phi\right) .
$$

Substituting (12) into (9)-(11), then one can obtain a characteristic equation for $\gamma$ :

$$
\begin{aligned}
\gamma^{3}+ & {\left[\frac{1}{\tau_{R}}+\frac{2 \kappa A}{\tau_{\text {in }}} \cos \left(\omega_{s} \tau\right)\right] \gamma^{2}+\omega_{R}^{2} \gamma } \\
+ & \frac{\kappa A}{\tau_{\text {in }}} \omega_{R}^{2}\left[\cos \left(\omega_{s} \tau\right)-\alpha \sin \left(\omega_{s} \tau\right)\right]=0
\end{aligned}
$$

with $A=1-\exp (-\gamma \tau), \tau_{R}^{-1}=\tau_{s}^{-1}+G_{N} E_{s}^{2}$, and $\omega_{R}^{2}=$ $G_{N} E_{s}^{2} / \tau_{p}$. The above equation is called the characteristic equation or the eigenvalue equation of the model. Each solution, which is also called the linear mode, predicts how the small perturbation to the initial steady state evolves with respect to time development.

\section{Chaos Control Via High-Frequency Modulation}

By numerically calculating (13), we obtain the linear mode solutions for a certain parameter condition. Two typical parameter conditions where chaotic output emerges are considered. One is the same with Fig. $2(\mathrm{c}): J=1.2 J_{\mathrm{th}}, L=9 \mathrm{~cm}$, and $r_{1}=2 \%$, the other is for $J=1.3 J_{\mathrm{th}}, L=15 \mathrm{~cm}$, 

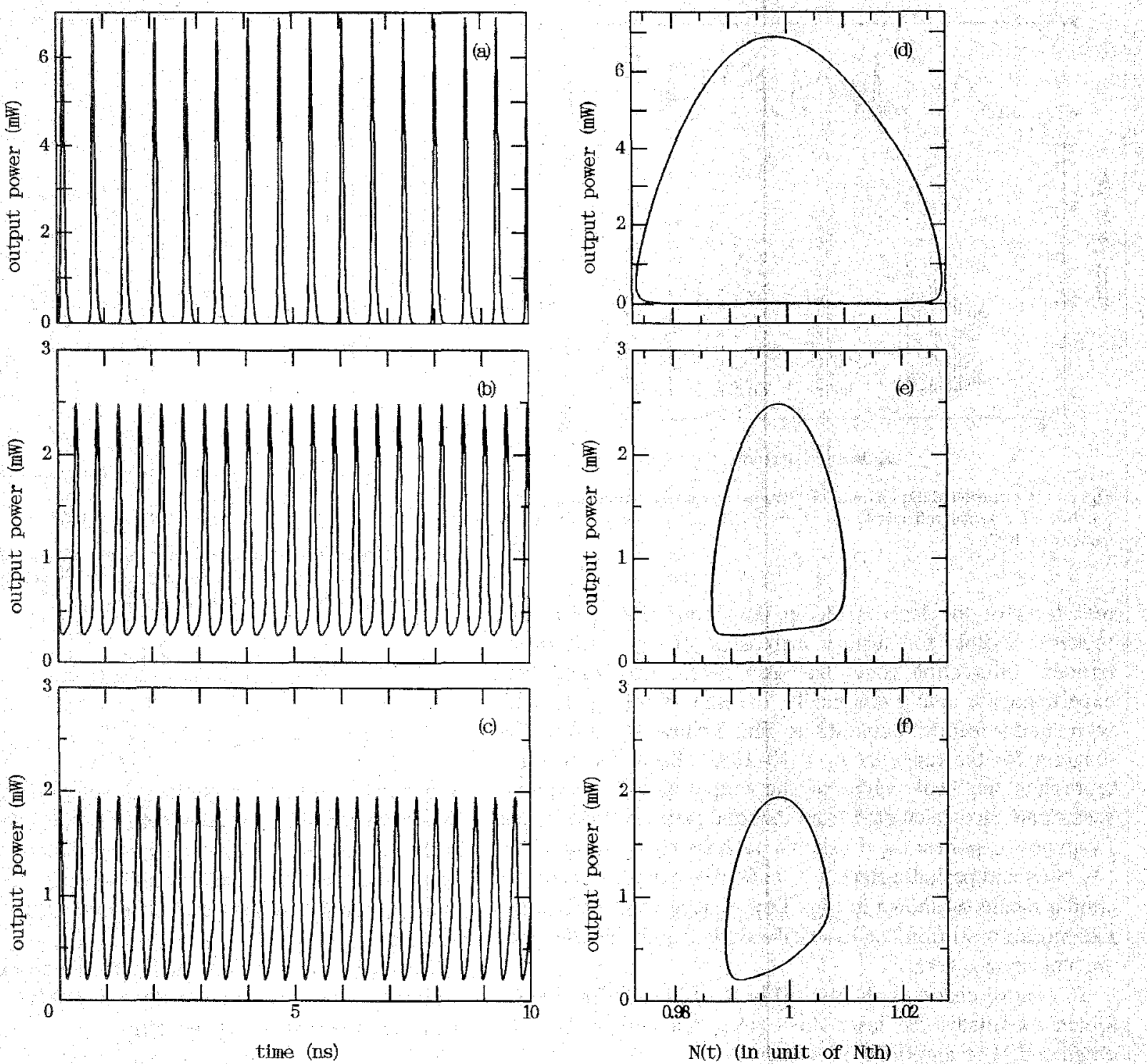

Fig. 5. Results of time series and phase portrait for $J=1.2 J_{\mathrm{th}}, L=9 \mathrm{~cm}$, and $r_{1}=2 \%$ : (a) and (d) $\nu=154 \mathrm{GHz}(\mathrm{arrow} \mathrm{A}$ in Fig, 4 ), (b) and (e) $\nu=2.19 \mathrm{GHz}$ (arrow B in Fig. 4), (c) and (f) $\nu=2.38 \mathrm{GHz}$ (arrow C in Fig. 4). The modulation depth $\xi$ is 0.1 .

and $r_{1}=2.5 \%$. Fig. 4 shows distribution of those modes whose frequencies are within 0.8 to $3.0 \mathrm{GHz}$. Two special cases are listed here to compare with our results. For a long distance external optical feedback, such as discussed in our previous paper [5], the modes distribute in a relatively regular way and the average separation between adjacent modes on the frequency axis is close to the inverse of the delay time, i.e., the external-cavity round-trip time. For a strong feedback level $\left(r_{1}=30 \%-45 \%\right)$, the linear modes distribute around the external-cavity mode and its harmonics, and the laser output also shows strong spectral peak at the cavity mode frequency, which is consistent with results reported in other experiments [13]. Whereas in the present paper, we only consider a short distance external feedback since we are interested in practical situations such like optical data recording system where the short distance and low level feedback is more likely to occur. In such circumstances, the delay time (usually less than $1 \mathrm{~ns}$ ) is near the relaxation time of the laser and the modes have a rather complicated distribution. For each mode, the real part shows the stability and the imaginary part determines the oscillation frequency The relevance between the linear mode frequency and the intrinsic resonant frequency of the dynamical system has been studied and utilized to explain the dynamical phenomena, the control of chaos, and accessing periodic oscillations in the previous researches [18]-[21]. So one may naturally raise a question whether such mode frequencies can be used as the modulation frequency to stabilize chaos with a small modulation depth. In the following, we show that the feedbackinduced chaos can be stabilized by modulating the injection current of the laser diode with frequencies chosen to be at or near the imaginary parts of those modes shown in Fig. 4

Fig. 5 shows results of modulations for $J=1.2 J_{\mathrm{th}}, L=9$ $\mathrm{cm}$, and $r_{1}=2 \%$. The original chaotic output without the modulation was shown in Fig, 2(c), Fig, 5(a), (b), and (c) shows temporal variations with the modulation frequencies of 

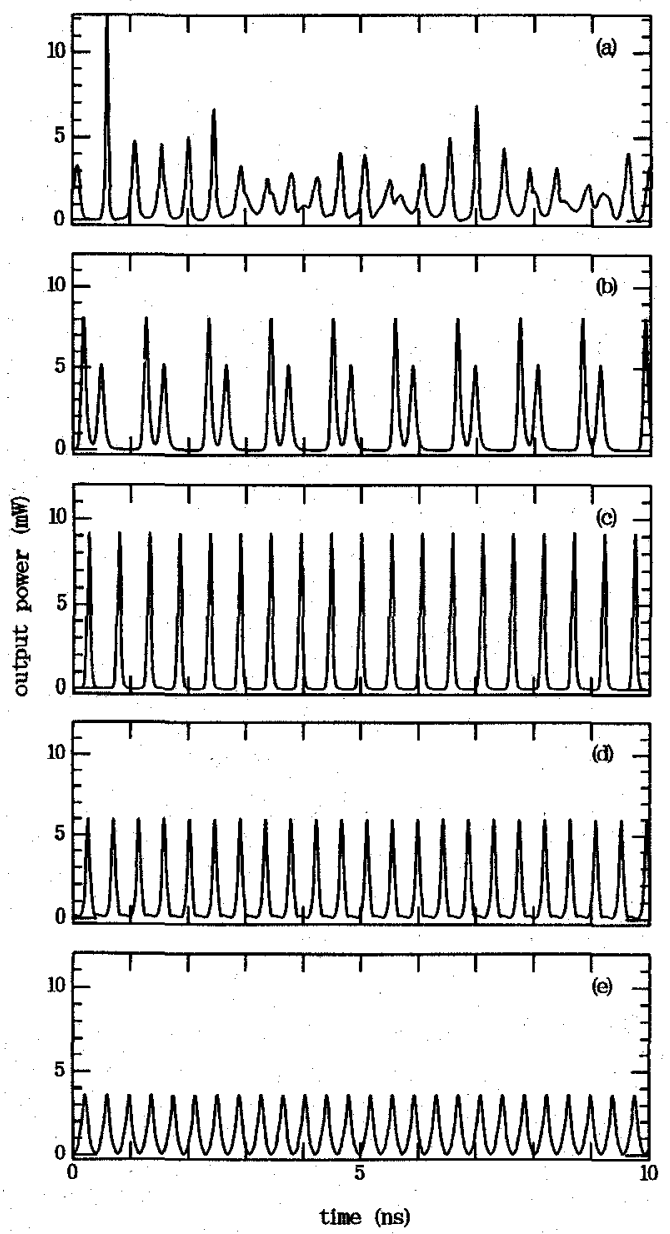

Fig. 6. Results of time series for $J=1.3 J_{\mathrm{th}}, L=15 \mathrm{~cm}$, and $r_{\mathrm{I}}=2.5 \%$ : (a) Without modulation, (b) $\xi=0.25, \nu=0.93 \mathrm{GHz}$ (arrow D in Fig. 4), (c) $\xi=0.15, \nu=1.90 \mathrm{GHz}$ (arrow $\mathrm{E}$ in Fig. 4), (d) $\xi=0.15, \nu=2.27 \mathrm{GHz}$ (arrow F in Fig. 4), (f) $\xi=0.15, \nu=2.62 \mathrm{GHz}$ (arrow G in Fig, 4).

$1.53,2.19$, and $2.38 \mathrm{GHz}$, which are equal to the frequencies of the modes in Fig. 4 indicated with arrows A, B, and C, respectively. From both the time series waveforms and the corresponding phase portraits [Fig. 5(d), (e), and (f)], it is clear that the chaotic output is stabilized to different limit cycles corresponding to different linear modes. There is a large increase of the oscillation amplitude in Fig. 5(a) compared with the original chaotic output of Fig. 2(c). This indicates that a strong resonant state exists around the frequency of 1.53 GHz. The modulation depth is $10 \%$ with respect to the bias injection current of the laser diode for all cases. Noting that the bias injection current is always above the threshold with the modulation depth of $10 \%$. Obviously, the feedback-induced chaos is stabilized to a limit cycle by modulating the laser diode injection current with the modulation frequency optimized by the linear stability analysis, although the modulation amplitude does not exceed the lasing threshold.

Another example of the modulation is demonstrated in Fig. 6 for $J=1.3 J_{\mathrm{th}}, L=15 \mathrm{~cm}$, and $r_{1}=2.5 \%$. The original chaotic output waveform is also plotted in Fig. 6(a) for comparison. Here, all of four modes shown in Fig. 4 (with black circles) are selected to modulate the laser diode. The modulation depth is carefully chosen to get the stable periodic

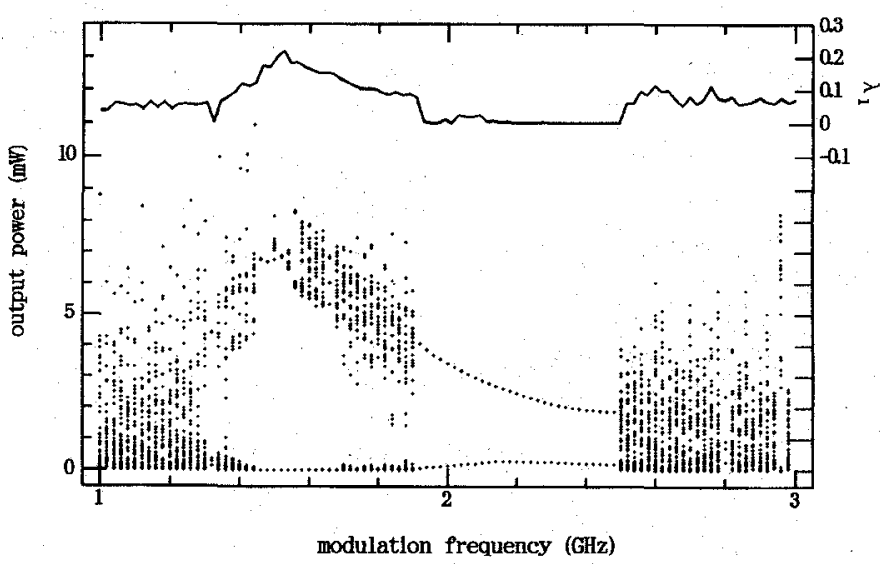

(a)

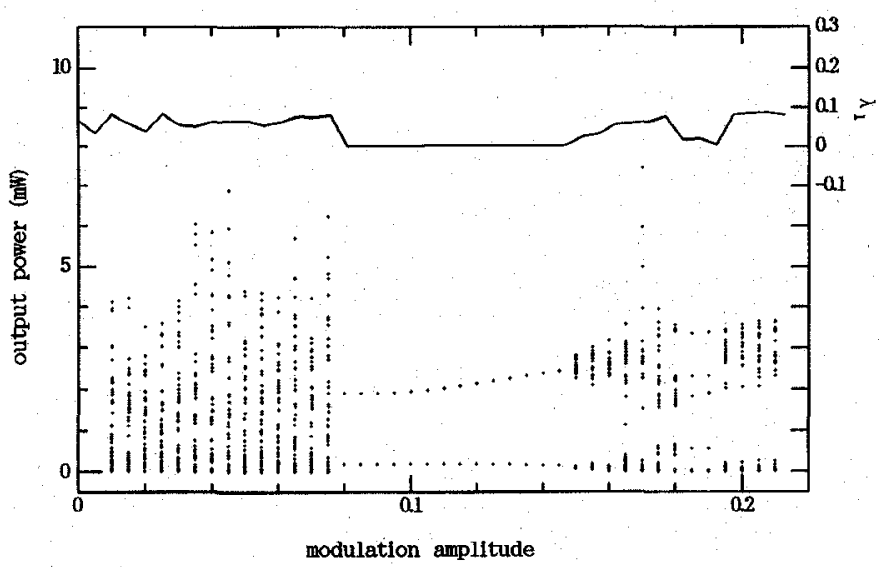

(b)

Fig. 7. Bifurcation diagrams and the largest Lyapunov exponent $\lambda_{1}$ as a function of modulation frequency and depth for $J=1.2 J_{\mathrm{th}}, L=9 \mathrm{~cm}$, and $r_{1}=2 \%$ : (a) Bifurcation versus modulation frequency at $\xi=0.1$, (b) Bifurcation versus modulation frequency at $\nu=2.38 \mathrm{GHz}$.

oscillations. Although the modulation depth of Fig. 6(b) is a little large (yet the bias injection is still above the threshold), the above results show that the feedback-induced chaos of the laser diode can be stabilized by direct modulations with a relatively small modulation depth if the modulation frequency is chosen to be equal to a linear mode frequency.

We studied the robustness of the modulation with respect to the modulation frequency and depth. For each linear mode chosen as the modulation frequency, we vary the depth and frequency around it and investigate the output variations. Fig. 7(a) and (b) shows bifurcation diagrams that are obtained by recording the peak values of the output power when the modulation frequency or depth is varied. The parameter values are $J=1.2 J_{\mathrm{th}}, L=9 \mathrm{~cm}$, and $r_{1}=2 \%$. Here, the largest Lyapunov exponent $\lambda_{1}$ is also shown as a function of the modulation frequency and depth. Fig. 7(a) shows the influence of the modulation frequency when it is changed from 1 to $3 \mathrm{GHz}$. The modulation depth is chosen to be 0.1 . Chaos is stabilized to periodic oscillations within two frequency domains which include all of three modes shown as white circles in Fig. 4. Fig. 7(b) shows the influence of the modulation depth which is changed from $1 \%$ to $21 \%$ at the modulation frequency of $2.38 \mathrm{GHz}$. Chaos is effectively 


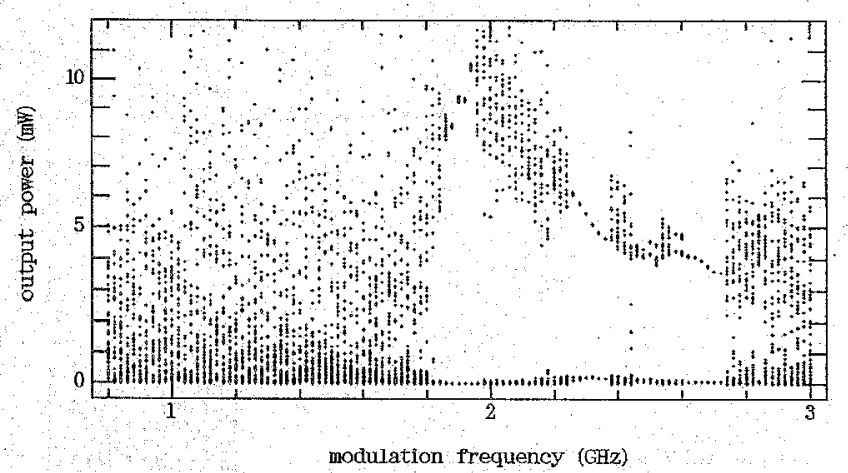

(a)

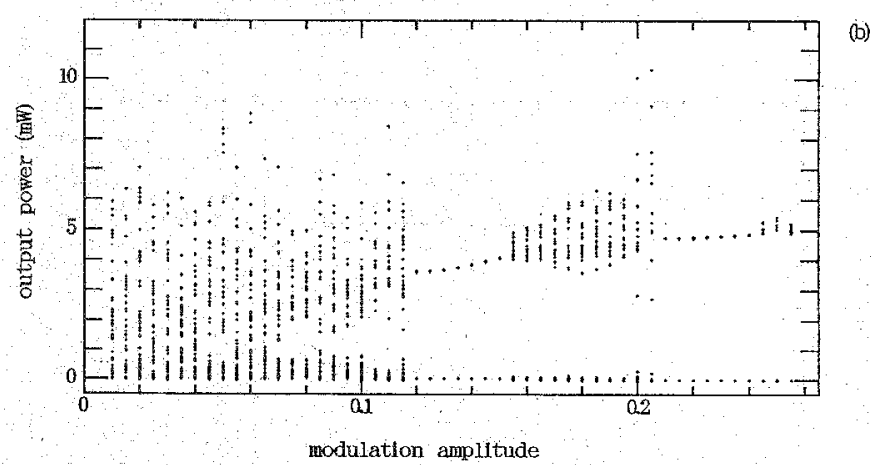

(b)

Fig. 8. Bifureation diagrams for $J=1.3 J_{\mathrm{th}}, L=15 \mathrm{~cm}$, and $r_{1}=2.5 \%$. (a) Bifurcation versus modulation frequency at $\xi=0.15$. (b) Bifurcation. versus modulation frequency at $\nu=2.62 \mathrm{GHz}$.

stabilized when the modulation depth takes the value between $8 \%$ to $14.5 \%$. The further increase of the modulation depth destroys periodic oscillations:

Fig. 8 shows another example of bifurcation diagrams for $J=1.3 J_{\mathrm{th}}, L=15 \mathrm{~cm}$, and $r_{1}=2.5 \%$. In Fig. $8(\mathrm{a})$, there exist several "windows" where the feedback-induced chaos is stabilized to limit cycles. One can easily verify that such "windows" contains the frequencies of the modes $E, F$, and $G$ in Fig. 4. The mode $D$ does not work at the modulation depth of 0.15 . The influence of the modulation depth is investigated in Fig. 8(b) for the modulation frequency of $2.62 \mathrm{GHz}$. Chaos is successfully controlled within two domains: $12 \%-15 \%$ and $21 \%-24 \%$. For modulation depths between these two domains, the original chaos reduces to quasi-periodic oscillations. Here, once again too large modulation depths fail to stabilize the chaos.

Modulation frequencies are essential in suppressing the feedback-induced chaos through injection modulation. We like to make a comparison of our results with those of [4]. There is a substantial difference between our work and [4]. We handled the problem for weak modulation while the latter dealt with strong modulation where the laser was forced to go below threshold during each modulation cycle. Similar conclusion about the modulation frequency has been obtained from our simple model and from the multimode model in [4]. However, in [4], the authors indicated that the optimized modulation frequency lay within the range of $\left(f_{\text {ext }} / 2, f_{\text {exi }}\right)$ for short

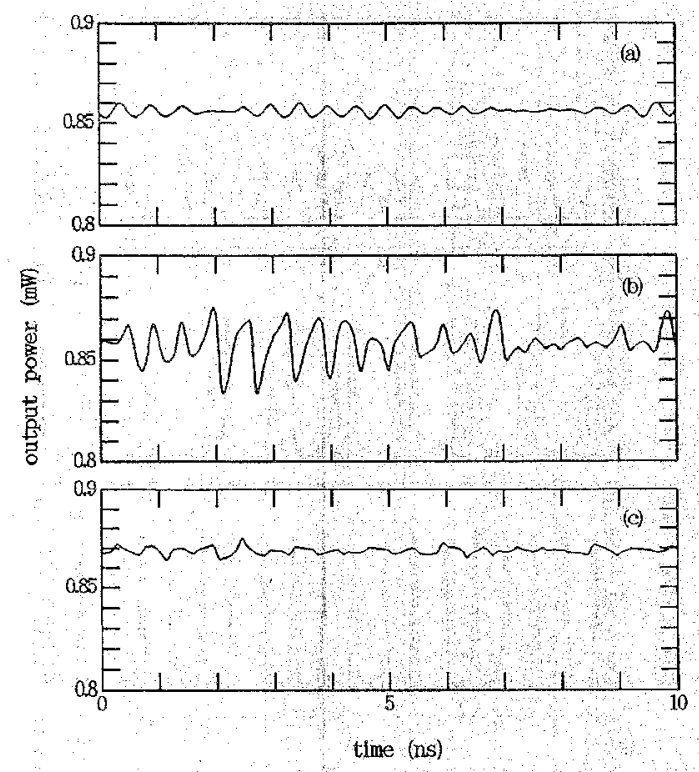

Fig. 9. 10-ns averaged time series for: (a) solitary laser, (b) laser with the external feedback $r_{1}=2 \%$ but without modulation, and (c) laser with both the external feedback $r_{1}=2 \%$ and the modulation $\xi=01, \nu=238 \mathrm{GHz}$ $J=1.2 J_{\mathrm{th}}, L=9 \mathrm{~cm}$ Langevin noises are noluded in the model.

cavities. The corresponding values of $f_{\text {ext }}$ in our simulations are to $1.67 \mathrm{GHz}$ and $1.0 \mathrm{GHz}$ for $L=9 \mathrm{~cm}$ and $15 \mathrm{~cm}$, respectively. We note that the primary modes for $L=9 \mathrm{~cm}$ [Fig. $5(\mathrm{a})]$ and $\mathrm{L}=15 \mathrm{~cm}[\mathrm{Fig}, 6(\mathrm{a})]$ read 1.54 and $0.93 \mathrm{GHz}$, respectively, and agree very well with those experimental and empirical results in [4]. The fact also verifies that the simple single-mode laser model can give good results for the laser diode even under weak modulations and feedbacks.

\section{SUPPRESSION OF FEEDBACK-INDUCED INTENSTTY NOISE}

We have so far neglected the role of spontaneous emission in order not to mix the effects of deterministic chaos with the stochastic noise. In practice, however, stochastic noise is always present in semiconductor lasers because of spontaneous emission and shot noise (resulting from the generation and recombination of charge cariers). These noise sources can be readily included in the rate equation through the Langevin noise terms $F_{e}(t), F_{\phi}(t)$, and $F_{N}(t)$. In this section, we study feedback-induced chaotic dynamics by including stochastic noise with emphasis on the variations of intensity noise level under different external feedback and nodulation conditions. The noise levels are determined from the following autocor relation relations [16]:

$$
\begin{aligned}
& \left\langle F_{e}(t) F_{e}(t+u)\right\rangle=\left\langle F_{\phi}(t) F_{\phi}(t+u)\right\rangle=\frac{1+X}{4 \tau_{p}} \delta(u) \\
& \left\langle F_{N}(t) F_{N}(t+u)\right\rangle=V-1\left(J+\frac{N_{0}}{\tau_{s}}+\frac{\chi E_{s}^{2}}{\tau_{p}}\right) \delta(u)
\end{aligned}
$$

where $\langle$ denotes a time average, $\delta$ is the delta function, $V$ is the volume of the active region, and $\chi$ is a constant involved in the emission and absorption processes whose value is in general 3 to 5 . 


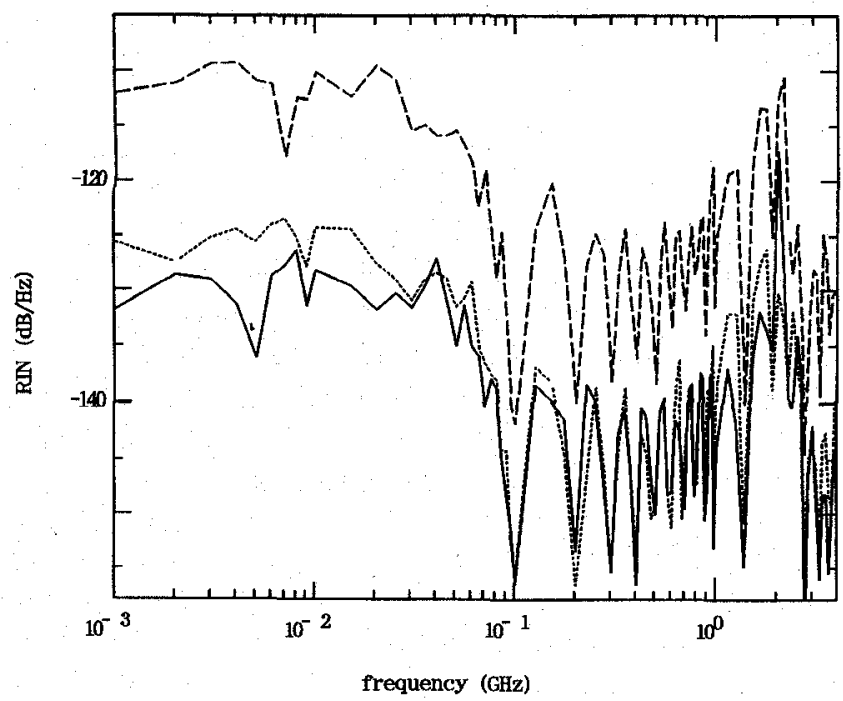

Fig. 10. RIN spectra corresponding to Fig. 9. Solid line: solitary laser [Fig. 9(a)]; Broken line: laser with the external feedback [Fig. 9(b)]; Dotted line: laser with the external feedback and the modulation [Fig. 9(c)].

Usually, RIN is employed to evaluate the noise characteristics of a laser diode. In the numerical calculations, the RIN can be obtained by calculating the spectrum of intensity fluctuations. Specifically, if $P(t)=\left|E_{0}(t)\right|^{2}$ is the optical power and $\bar{P}$ is its average value, the RIN spectrum is defined as the Fourier transform of the autocorrelation function according to the relations

$$
\begin{aligned}
& S(\omega)=\int_{-\infty}^{\infty}\left\langle\delta P(t) \delta P\left(t+t^{\prime}\right)\right\rangle \exp \left(-i \omega t^{\prime}\right) d t^{\prime} \\
& \text { RIN }=\frac{S(\omega)}{\bar{P}^{2} \cdot \Delta f}
\end{aligned}
$$

where $\delta P(t)=P(t)-\bar{P}$ is the fluctuation at time $t$.

When both the external feedback and Langevin noises are considered, the output time series exhibit stochastic fluctuations as well as chaotic variations. In this paper, the attention is paid to low-frequency fluctuations since the bandwidth of the actual optical data recording system generally does not exceed $100 \mathrm{MHz}$. Therefore, it is beneficial to remove the highfrequency fluctuation in the output time series by performing a running average over a 10-ns square window. The resulting time series becomes a measure of low-frequency fluctuations and reflects how a typical time-resolved spectrum of the laser diode would appear in actual experiments where generally a detector with the band width of 100 to several ten $\mathrm{MHz}$ is used to detect the light output of the laser diode. Fig. 9(a) and (b) shows the temporal variations of averaged outputs for a solitary laser and the same laser with external feedback, respectively. The output power of the solitary laser is nearly constant, while it exhibits large fluctuations in the presence of external feedback. Such low-frequency fluctuations are indicative of feedback-induced chaos. On the other hand, when the modulation is applied, the low-frequency fluctuation is greatly reduced as shown in Fig. 9(c). In fact, the lowfrequency fluctuation level in Fig. 9(c) is almost the same with that of the solitary laser.

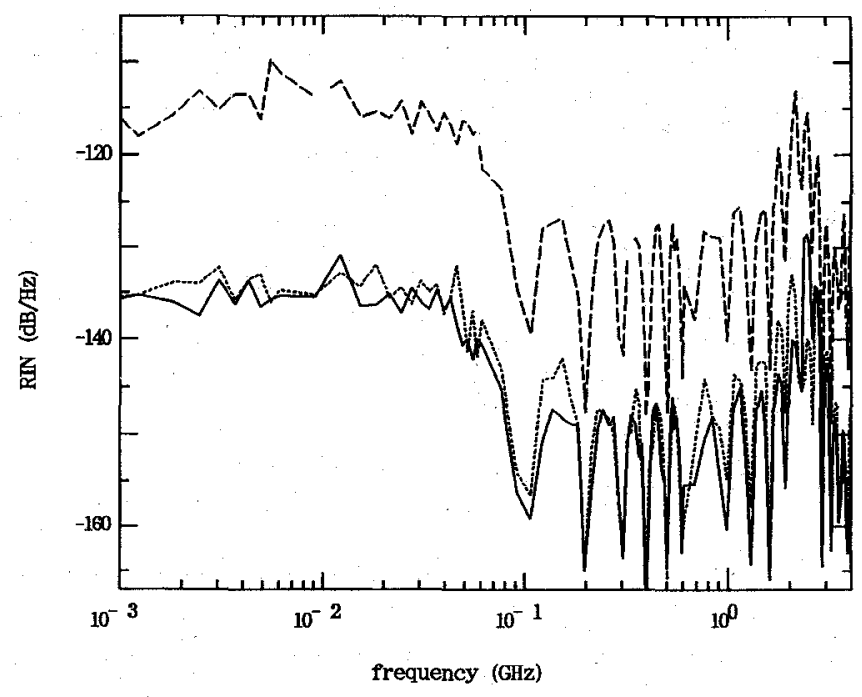

Fig. 11. RIN spectra for $J=1.3 J_{\mathrm{th}}$ and $L=15 \mathrm{~cm}$. Solid line: solitary laser; Broken line: laser with the external feedback $r_{1}=2.5 \%$; Dotted line: laser with the external feedback $r_{1}=2.5 \%$ and the modulation $\xi=0.15, \nu=$ $2.62 \mathrm{GHz}$.

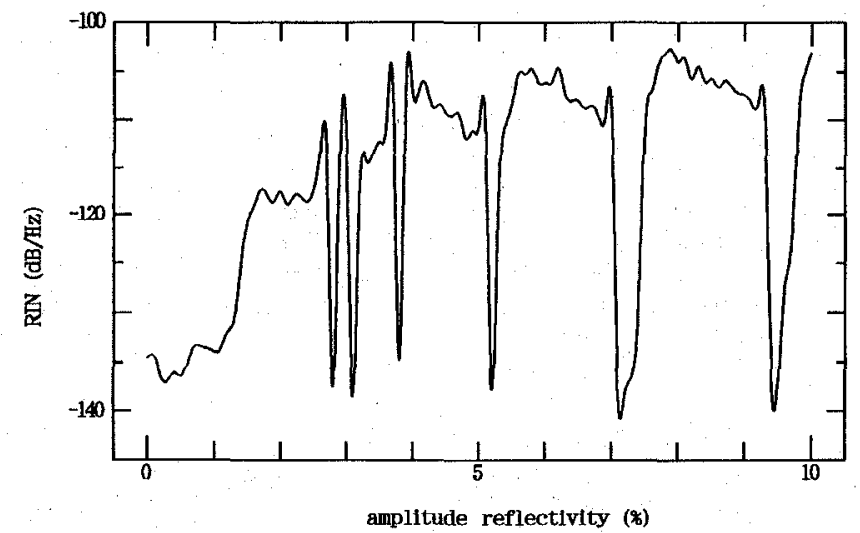

Fig. 12. Variation of low-frequency RIN (averaged over 0 to $100 \mathrm{MHz}$ ) versus feedback reflectivity for $J=1.2 J_{\mathrm{th}}, L=9 \mathrm{~cm}$.

We calculated the RIN based on (17) and show the RIN spectra in Figs. 10 and 11. The solid, broken, and dotted lines in Fig. 10 show the RIN spectra corresponding to Fig. 9(a), (b), and (c), respectively. Fig. 11 shows the RIN spectra of another parameter condition. The solid, broken, and dotted lines also correspond to the solitary laser, the laser with the external optical feedback, and the laser with both the feedback and the modulation, respectively. One easily finds that the low-frequency RIN is enhanced by a large amount (15-20 dB) because of the external feedback and such enhancement is suppressed to the level of the solitary laser when the feedbackinduced chaos is stabilized through the injection modulation. High-frequency peaks corresponding to the relaxation oscillation and the modulation frequencies are also recognized in these figures.

To get a further insight into the relation between the RIN level and deterministic chaos, we investigate bifurcation diagrams of the RIN level versus the reflectivity and the modulation parameters. Fig. 12 shows the variation of the lowfrequency RIN (averaged over the $0-100-\mathrm{MHz}$ range) as a 


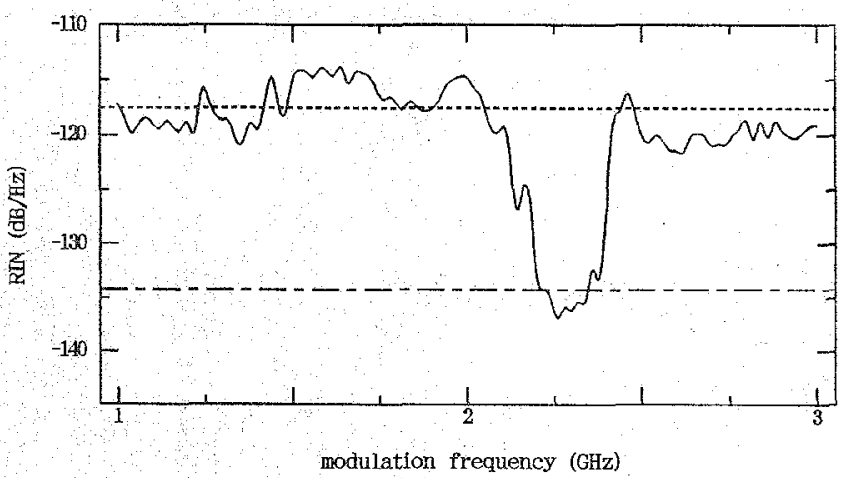

(a)

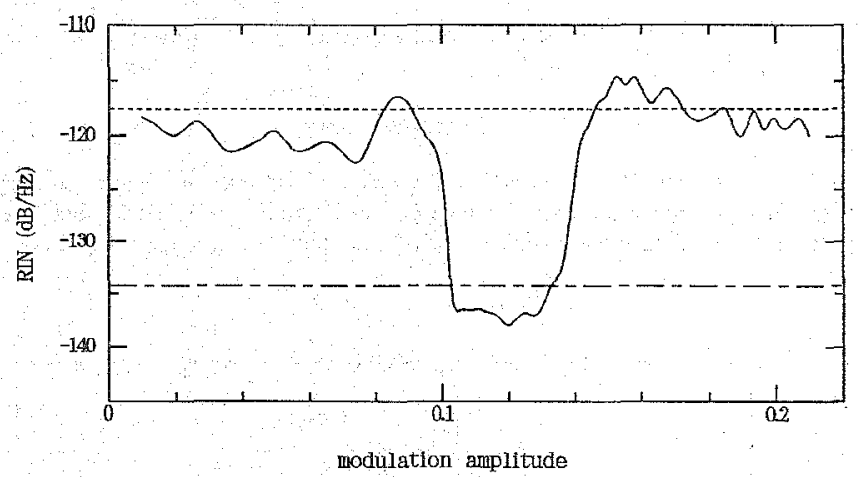

(b)

Fig. 13. Variation of low-frequency RIN (averaged over 0 to $100 \mathrm{MHz}$ ) versus (a) modulation frequency and (b) modulation depth. Parameter condition is the same as that in Fig. 7. The dashed straight lines indicate the RIN level for the solitary laser and the dotted lines indicate that for the laser with the external feedback but without the modulation.

function of the feedback reflectivity $r_{1}$. The corresponding bifurcation diagram without stochastic noise was shown in Fig. 3. There is one-to-one correspondence between the regions of chaos and the regions of high-RIN level. This clearly demonstrates that deterministic chaos is the cause of highRIN levels observed in semiconductor lasers in the presence of optical feedback. The low-frequency RIN is plotted in Fig. 13(a) as a function of the modulation frequency and also in Fig. 13(b) as a function of the modulation depth. The parameter condition is the same as that of Fig. 7. By comparing Fig. 13 with Fig. 7, it can be concluded that the RIN level decreases only in the region where the feedbackinduced chaos is stabilized to limit cycles. We note that the stabilization of the feedback-induced chaos does not always mean the reduction of the RIN. Particularly, the modulations which cause very strong amplifications of the oscillation amplitude such like frequency domains around $1.5 \mathrm{GHz}$ in Fig. 7(a) or $19 \mathrm{GHz}$ in Fig. 8(a), rarely result in the reduction of the RIN level. This is because the stabilization with that modulation frequency is very fragile to noise. In fact, when the Langevin noises are included in the model, the counterparts of Figs. $5(\mathrm{c})$ and $6(\mathrm{~d})$ remain periodic oscillations with small fluctuations in the waveforms, while those of Figs. 5(a) and 6(b) turn out to be chaotic. So the low-frequency fluctuations of the latter do not decrease as it did in the former cases. Therefore, only the stabilization with the robust structure to noise can result in the reduction of the low-frequency RIN level.

\section{CONCLUSION}

We have investigated the elimination or suppression of chaotic behavior in a semiconductor laser with external optical feedback. We have considered that the RIN enhancement is explained by coherence collapse and bifurcation to chaos due to the external optical feedback. The feedback-induced intensity noise has been suppressed by a high-frequency modulation technique, in which the modulation frequency is chosen to be equal to or near the mode frequencies obtained from the linear stability analysis. The RIN at low-frequency region has been reduced to the solitary laser level by the optimized modulation frequency. We have also investigated the robustness of the chaos control against the modulation frequency and the depth around the linear modes.

In [4, Figs. 17 and 18$]$ and $[2$, Figs, 2 and 5$]$, the authors have experimentally investigated feedback-induced noise enhancement and noise suppression via HFI in semiconductor lasers. Their results indicated that there exist certain frequencies where the RIN level is largely decreased compared with other frequencies. Also in their works, the robustness of mod ulation frequency and depth were experimentally examined. This paper handled the noise suppression problem from the chaos-controlling viewpoint and gave an analytical method for determining the optimum modulation for HFI technique. Our numerical results, Fig. 13 (also Figs. 7 and 8), show same inclinations with what reported in [4] and [2]. Besides externalcavity semiconductor lasers, chaos stabilization through direct periodic modulation or parametric perturbation has also been experimentally investigated in other systems [6], [7], [20], where it has been suggested that effective frequencies for the modulation might be resonant frequency or its harmonics. In this paper, the linear stability analysis has just given an analytical estimation for such resonant frequency of the external-cavity laser diode

Chaos control has been performed in various systems in the past few years. The concept of control chaos" means the stabilization of the unstable periodic orbits (UPO s) which are originally embedded in the chaotic attractor without dramat ically changing the system configuration [22]. Such UPO'S can be obtained from the chaotic attractors which might be reconstructed from the time series. The OGY method gives a strict algorithm to control one specific UPO, while the OPF method prefers to stabilize one of existent UPO's [23], [24]. The distribution of UPO's exhibits some peaks at certain frequencies So the optimization of the modulation frequency in stabilizing chaos through the direct modulation can be viewed as a problem to look for the most appropriate frequency where the UPO's are most likely to locate. As discussed in our previous works, the linear mode indeed revealed resonant features of the dynamical system. Therefore, driving the system at such mode frequencies can stabilize the chaos to limit cycles even with a small modulation depth. However, when the modulation depth is very large, the modulation itself dramatically changes the dynamical property 
of the control target and the mechanism, of course, belongs to a different category.

\section{REFERENCES}

[1] D. Lenstra, B. H. Verbeek, and A. J. den Boef, "Coherence collapse in single-mode semiconductor lasers due to optical feedback," IEEE J. Quantum Electron, vol. QE-21, pp. 674-679, 1985.

[2] A. Arimoto, M. Ojima, N. Chinone, A. Oishi, T. Gotoh, and N. Ohnuki, "Optimum conditions for the high frequency noise reduction method in optical videodisk system," Appl. Opt., vol. 25, pp. 1398-1403, 1986.

[3] T. Kanada, "Theoretical study of noise reduction effects by superimposed pulse modulation," Trans. IECE Jpn., vol, E-68, pp. 180-187, 1985.

[4] A. T. Ryan, G. P. Agrawal, G. R. Gray, and E. C. Gage, "Opticalfeedback-induced chaos and its control in multimodal semiconductor lasers," IEEE J. Quantum Electron., vol. 30, pp. 668-679, 1994.

[5] Y. Liu, N. Kikuchi, and J. Ohtsubo, "Controlling dynamical behavior of a semiconductor laser with external optical feedback," Phys. Rev. E, vol. 51, pp. R2697-2700, 1995.

[6] A. Azevedo and S. M. Rezende, "Controlling chaos in spin-wave instabilities," Phys. Rev. Lett., vol. 66, pp. 1342-1345, 1991.

[7] L. Fronzoni, M. Giocondo, and M. Pettini, "Experimental evidence of suppression of chaos by resonant parametric perturbations," Phys. Rev. A, vol. 45 , pp. 6483-6487, 1991 .

[8] R. ChacÛn and J. D. Bejarano, "Routes to suppressing chaos by weak periodic perturbation," Phys. Rev. Lett., vol. 71, pp. 3103-3106, 1993.

[9] R. Meucci, W. Gadomski, M. Giofini, and F. T. Arecchi, "Experimental control of chaos by means of weak parametric perturbations," Phys. Rev. $E$, vol. 49 , pp. R2528-R2581, 1994.

[10] N. Watanabe and K. Karaki, "Inducing periodic oscillations from chaotic oscillations of a compound-cavity laser diode with sinusoidally modulated drive," Opt. Lett., vol. 20, pp. 1032-1034, 1995.

[11] J. Mørk, B. Tromborg, and J. Mark, "Chaos in semiconductor lasers with optical feedback: Theory and experiment," IEEE J. Quantum Electron., vol. 28 , pp. 93-108, 1992.

[12] H. Li, J. Ye, and J. G. McInerney, "Detailed analysis of coherence collapse in semiconductor lasers," IEEE J. Quantum Electron., vol. 29, pp. 2421-2432, 1993.

[13] T. Mukai and K. Ohtsuka, "New route to optical chaos: Successivesubharmonic-oscillation cascade in a semiconductor laser coupled to án external cavity," Phys. Rev. Lett., vol. 55, pp. 1711-1714, 1985.

[14] B. C. Lam, M. M. Sushchik, H. D. I. Abarbanel, A. L. Keller, and P. K. L. Yu, "Relaxation-oscillation-induced chaotic instabilities in modulated external-cavity semiconductor lasers," J. Opt. Soc. Amer. B, vol. 12, pp. $1150-1156,1995$.

[15] R. Lang and $\mathbf{K}$. Kobayashi, "External optical feedback effects on semiconductor injection laser properties," IEEE J. Quantum Electron., vol. QE-16, pp. 347-355, 1980

[16] C. H. Henry, "Theory of spontaneous emission noise in open resonators and its application to lasers and optical amplifiers," J. Lightwave Technol., vol. LT-4, pp. 288-297, 1986.

[17] B. Tromborg, J. H. Osmundsen, and H. Olesen, "Stability analysis for a semiconductor laser in an external cavity," IEEE J. Quantum Electron, vol. QE-20, pp. 1023-1032, 1984.

[18] R. VallËe and C. Delisle, "Mode description of the dynamical evolution of an acousto-optic bistable device," IEEE J. Quantum Electron, vol. QE-21, pp. 1423-1428, 1985.
[19] Y. Liu, J. Ohtsubo, and Y. Shoji, "Accessing high-mode oscillations in a delayed optical bistable system," Opt: Commun, vol. 105, pp. 193-198, 1994.

[20] Y: Liu and J. Ohtsubo, "Experimental control of chaos in a laser-diode interferometer with delayed feedback," Opt. Lett., vol. 19, pp. 448-450, 1994

[21] Y. Liu and J. Ohtsubo, "Controlling chaos of a delayed optical bistable system," Opt. Rev., vol. 1, pp. 91-93, 1994

[22] E. Ott, C. Grebogi, and J. A. Yorke, "Controlling chaos," Phys. Rev. Lett., vol. 64, pp. 1196-1199, 1990.

[23] E. R. Hunt, "Stabilizing high-period orbits in a chaotic system: The diode resonator," Phys. Rev. Lett., vol. 67, pp. 1953-1956, 1991.

[24] R. Roy, T. W. Murphy, T. D. Maier, Z. Gills, and E. R. Hunt, "Dynamical control of a chaotic laser: Experimental stabilization of a globally coupled system," Phys. Rev. Lett., vol. 68, pp. 1259-1262, 1992.

Noriyuki Kikuchi was born in Shizuoka, Japan, in 1972. He received the B.S. degree in optoelectronics and mechanical engineering in 1994. He is currently working towards the masters degree at Shizuoka University, Hamamatsu, Japan.

His research interest is nonlinear dynamics in semiconductor lasers.

Mr. Kikuchi is a member of the Japan Society of Applied Physics.

Yun Liu was born in 1964 in Anhui, China. He received the B.S. degree in mechanics from the University of Science and Technology of China, Hefei, China, in 1986, and the M.S. and Ph.D. degrees in electronics, both from Shizuoka University, Hamamatsu, Japan, in 1991 and 1994, respectively.

Currently, he is a Research Associate at Shizuoka University. His research interests include nonlinear dynamics in optics, semiconductor lasers, and neural networks.

Dr. Liu is a member of the Optical Society of America and the Japan Society of Applied Physics.

Junji Ohtsubo (M'95) received the B.S. degree in electronics from the Kyushu Institute of Technology in 1973 and the M.S. and Ph.D. degrees in electronics from Hokkaido University in 1975 and 1978, respectively.

In 1978, he joined the Mechanical Engineering Laboratory. During 1981-1982, he was a Research Associate at the Institute of Optics, University of Rochester, Rochester, NY. He joined Shizuoka University, Hamamatsu, Japan, as an Associate Professor in 1985 and is presently a Professor. His current research interests are statistical optics, speckle, optical metrology, active interferometer, optical information processing and computing, and nonlinear dynamics in optics.

Prof. Ohtsubo is a member of the Optical Society of America, SPIE, the Japan Society of Applied Physics, the Optical Society of Japan, and the Laser Society of Japan. 